obstruction. Emergency colorectal surgery is associated with high morbidity and mortality rates.

Preoperative decompression (SEMS or trananal decompression tube placement) as a bridge to surgery has been reported to improve short-term outcomes. However, oncological safety of endoscopic interventions is debatable due to the high frequency of perforations and dissemination. There are few retrospective studies of comparative outcomes between SEMS and decompression tube, but the benefits were not clear. The aim of this prospective randomized study is to compare sortterm and especially long term outcome of colorectal stenting and transanal decompression tube as a bridge to surgery.

Methods Between December 2012 and December 2017, 72 patients with malignant colon obstruction, which was diagnosed based on radiological and endoscopic findings with pathohistological examination, were consecutively included into the study. Patients were randomized of random sampling numbers and divided into the stent or decompression tube group.

Results Technical success rate RR 0.914, 95\% CI 0.804-1.039 $(p=0.065)$. Clinical success was significant higher in stent group RR 0.643 , 95\% CI $0.489-0.846(\mathrm{p}=0.031)$. Rate of complications was similar in groups RR 0.895 , 95\% CI $0.755-1.061 \quad(p=0.087)$. Short-term outcomes in SEMS group were superior to decompression tube group in the following areas: stoma creation rate RR 0.914 , 95\% CI $0.804-1.039$ $(p=0.065) \quad(p<0.01)$, need to ICU RR $0.673,95 \%$ CI $0.493-$ $0.919(\mathrm{p}<0.05)$, surgical infection rate RR 0.721 , 95\% CI $0.520-0.999(p<0.05)$. No significant differences were found in the overall postoperative complication rate $\mathrm{RR} 0.748,95 \%$ CI $0.535-1.044(\mathrm{p}=0.117)$, anastomotic leak rate RR 0.953, $95 \%$ CI $0.828-1.056(\mathrm{p}=0.062)$, duration of in-patient stay $17(10-21)$ and $20(14-25)$ days $(p=0.248)$. In long-term period rate of recurrence was significant higher in stent group $29.4 \%$ vs $17.8 \%(\mathrm{p}=0.031)$. However, the1-year overall survival rate $89.0 \%$ and $83.3 \%(\mathrm{p}=0.423)$, and 3 -year of patients $69.4 \%$ and $63.9 \%(\mathrm{p}=0.460)$, in the stent and tube groups, respectively, was not significant different.

Conclusions SEMS has some benefits in comparison with decompression tubes in the short-term postoperative period for patients with malignant colorectal obstruction. The overall recurrence rate was higher in the stent group in long-term period. Moreover, the overall survival rate in the group is no different. Need to continue research to material accumulation and analysis of long-term results.

\section{PTU-127 POOR QUALITY OF CAPSULE ENDOSCOPY IMAGES HAS NEGATIVE EFFECT ON DIAGNOSIS OF SMALL BOWEL MALIGNANCY}

Diana Yung*, Anastasios Koulaouzidis, John Plevris. The Royal Infirmary of Edinburgh, Edinburgh, UK

\subsection{6/gutjnl-2019-BSGAbstracts.116}

Introduction Capsule endoscopy (CE) is the prime mode of investigation for small bowel (SB) pathology. It relies heavily on image quality, which is commonly affected by poor preparation. Currently, there is no widely-accepted method for quantifying visualisation quality. We studied the contribution of various image parameters to visualisation quality and their effect on certainty of diagnosis of small bowel lesions.
Methods Five clear CE images of common SB pathology were processed for 3 parameters to simulate increasingly poor SB preparation: opacity (colour-matched to luminal content; 10 90\%, 10\% increments), blurriness (radius 1-10px; 1px increments), contrast $(-50-50 \% ; 10 \%$ increments). 9 expert readers evaluated whether images were adequate for diagnostic purposes. The points where perception of image quality changed significantly were determined for each parameter. Three further sets of SBCE images (vascular, inflammatory and malignant lesions) were processed for 4 points/parameter. 20 experienced-expert CE readers from reviewed the resulting images.

Results In vascular and inflammatory lesions, diagnostic certainty was least affected by increasing image opacity, requiring opacities $>90 \%$ before most readers considered images inadequate for diagnosis. The greatest negative effects of image opacity were seen in malignancy where significantly fewer readers found images adequate at $>50 \%$ opacity. Similar results obtained with increasing blur radius, simulating motion blur and poor focus. The proportions of readers finding vascular and inflammatory images adequate for diagnosis did not drop significantly at wider blur radii, while the proportion who found images of malignancies diagnostically adequate dropped at blur radius $6 \mathrm{px}$. Decreasing contrast had greater negative effect than raised contrast, most obvious in malignant lesions.

Conclusions Poor visualisation quality in all parameters had the greatest effect on malignant lesions. Software to increase contrast and sharpen images can improve visualisation quality; smart frame rate adaptation could also improve the number of high-quality frames obtained. Furthermore, our results suggest that thoroughness in SB cleansing is most important when there is suspicion of SB malignancy, to improve diagnostic certainty of images obtained.

\section{PTU-128 DOUBLE-HEADED CAPSULE ENDOSCOPY: REAL-WORLD EXPERIENCE FROM A MULTICENTRE BRITISH STUDY}

${ }^{1}$ Diana Yung*, 'Joanna Brzeszczynska, ${ }^{2}$ Imdadur Rahman, ${ }^{3}$ Leena Sinha, ${ }^{4}$ Reena Sidhu, ${ }^{2}$ Praful Patel, ${ }^{3}$ Sue Mason, ${ }^{4}$ Mark McAlindon, ${ }^{1} J o h n$ Plevris, ${ }^{1}$ Anastasios Koulaouzidis. ${ }^{1}$ The Royal Infirmary of Edinburgh, Edinburgh, UK; ' 2 Southampton General Hospital, Southampton, UK; ${ }^{3}$ Queen's Hospital, Romford, London, UK; ${ }^{4}$ Royal Hallamshire Hospital, Sheffield, UK

\subsection{6/gutjnl-2019-BSGAbstracts.117}

Introduction Capsule endoscopy (CE) is a well-established mode of investigation for small bowel (SB) pathology. This study examines the potential benefits of using double-headed capsules compared to conventional single-headed ones in a real-world cohort of patients referred for CE. We present initial results from the first multicentre British study.

Methods Over a 9-month period, patients referred for routine SBCE at 4 tertiary referral centres in the UK underwent double-headed CE in lieu of conventional single-headed CE using MiroCam MC2000 capsules. CE was carried out as per routine protocols at each centre. Clinical data were anonymised. One head $(\mathrm{L} / \mathrm{R})$ was chosen at random and reported by an expert reviewer. The double-headed recordings, also anonymised and randomised, were reported by another expert reviewer. In centres with only one expert reviewer, doubleheaded CEs were read after a 4-week interval to minimise recall bias. For each CE, numbers and types of findings and 


\begin{tabular}{|c|c|c|c|c|}
\hline Indication & $\begin{array}{l}\text { SB bleeding } \\
(n=94)\end{array}$ & $\begin{array}{l}\text { ?IBD/IBD reassessment } \\
(\mathrm{n}=84)\end{array}$ & $\begin{array}{l}\text { ?SB neoplasia } \\
(\mathrm{n}=15)\end{array}$ & $\begin{array}{l}\text { Others } \\
(n=11)\end{array}$ \\
\hline $\begin{array}{l}\text { No. of CEs where findings differed } \\
\text { between heads }\end{array}$ & $27(28.7 \%)$ & $30(35.7 \%)$ & $2(13.3 \%)$ & $1(9.1 \%)$ \\
\hline $\begin{array}{l}\text { Mean numerical difference in findings } \\
\text { between heads (range) }\end{array}$ & $3.4(0-16)$ & $4.3(0-39)$ & $4(3-5)$ & 3 \\
\hline $\begin{array}{l}\text { No. of CEs where differences were } \\
\text { clinically significant }\end{array}$ & $\begin{array}{l}17(18.1 \%) \\
\text { In } 1 \mathrm{CE} \text {, no. of findings was same but type was } \\
\text { significantly different }\end{array}$ & $\begin{array}{l}11(13.1 \%) \\
\text { In } 1 \mathrm{CE} \text {, no. of findings was same but type } \\
\text { was significantly different }\end{array}$ & $2(13.3 \%)$ & $0(0 \%)$ \\
\hline Details & $\begin{array}{l}\text { Findings missed by single-headed CE: } 16 \\
\text { 1. Angioectasias (5) } \\
\text {-SB inflammation (7) } \\
\text { - Oesophagitis (2) } \\
\text {-SB masses (2) } \\
\text { Difference in findings changed assessment of extent/ } \\
\text { severity: } 1 \text { (angioectasias) }\end{array}$ & $\begin{array}{l}\text { Missed findings: } 5 \\
\text { - In all, signs of active inflammation missed } \\
\text { by single-headed CE } \\
\text { Difference in findings changed assessment of } \\
\text { extent/severity: } 6\end{array}$ & $\begin{array}{l}\text { Missed findings: } 1 \text { polypoid mass, } \\
1 \text { SB diverticulum }\end{array}$ & na \\
\hline
\end{tabular}

overall conclusion/diagnosis were compared between single and double-headed examinations.

Results 211 CE examinations were performed. 7 failed to reach the SB; 204 cases were analysed. Indications were (a) SB bleeding; (b) ?SB inflammation or reassessment of known inflammatory bowel disease (IBD); (c) ?SB neoplasia including suspicious radiological imaging and (d) others e.g. ?coeliac disease. Results are presented in table 1 .

Overall, use of two CE heads impacted the diagnosis in 30/ $204(14.7 \%)$ of cases in our cohort.

Conclusions The use of double-headed CE provides more information which has the potential to change clinical diagnosis and therefore management. Therefore, the routine adoption of double-headed CE in SB assessment should be considered.

\section{PTU-129 INDICATIONS AND OUTCOMES OF COLONOSCOPY IN THE VERY ELDERLY PATIENTS}

Abdulkani Yusuf*, Ben Ayeboa-sallah, Mustafa Agbna, Khai Leow, Holly Tucker, Arun Jeevagan, Phil Mayhead. East Sussex Healthcare NHS Trust, Eastbourne, UK

\subsection{6/gutjnl-2019-BSGAbstracts. 118}

Introduction Colonoscopy is the investigation of choice for lower gastrointestinal symptoms. We wanted to review the indications and outcomes of colonoscopy in the very elderly patients at a district general hospital.

Methods We retrospectively reviewed all patients $\geq 85$ years who underwent colonoscopy from June 2016- June 2018using using our endoscopy reporting system (Endobase). We collected data on demographics, indications for the procedure, bowel preparation type and success, sedation and analgesia rates, caecal intubation rates, findings and complication rates.

Results From June 2016-June 2018, there were 243 patients $\geq 85$ years who underwent colonoscopy, mean age 87.0 years (range 85-96), males 41.8\%, females 51.9\%. The commonest indications were: change of bowel habit $(\mathrm{n}=75$, $30.1 \%)$, anaemia $(n=53,21.8 \%)$, abnormal imaging $(n=30$, $12.3 \%)$, rectal bleeding $(n=25,10.3 \%)$, polyp surveillance $(\mathrm{n}=18,7.4 \%)$, polypectomy $(\mathrm{n}=16,6.6 \%)$, colon cancer surveillance $(\mathrm{n}=10,4.1 \%)$, IBD surveillance $(\mathrm{n}=2$, $0.4 \%)$, and other $(n=9,3.7 \%)$. All patients went thorough bowel preparation with PEG solution with excellent or adequate results in 209 (86.0\%), and inadequate in 34 (14.0\%). Fentanyl was used in 175 patients $(72.0 \%$, average dose $43.2 \mathrm{mcg})$, Pethidine in $17(7.0 \%$, average dose 26.5 $\mathrm{mcg}$ ), Entonox in 27 patients (11.1\%), and Midazolam in 184 patients $(75.7 \%$, average dose $1.84 \mathrm{mg})$. Unadjusted caecal intubation rate was $84.4 \%(n=205)$, rising to $88.7 \%$ when adjusted for obstructing lesions. Commonest causes for incomplete procedure $(\mathrm{n}=38)$ were: Obstructing lesions $(\mathrm{n}=12,31.6 \%)$, inadequate bowel preparation $(\mathrm{n}=7$, $18.4 \%)$, patient intolerance $(n=6,15.7 \%)$, and diverticulosis $(n=5,13.2 \%)$. Cancer was found in 21 patients $(8.6 \%)$. Polyps of any size were found in 90 patients (37.0\%), but significant polyps (polyps $\geq 10 \mathrm{~mm}$ ) were found in 42 patients $(17.3 \%)$ with average polyp size of $20.7 \mathrm{~mm}$ (range 10-80 $\mathrm{mm})$. Other significant findings included colonic diverticulosis in $(n=147,60.5 \%)$, haemorrhoids $(n=73,30.0 \%)$, and IBD $(n=4,1.6 \%)$. Three patients developed peri-procedure complications: Oxygen desaturation and tachycardia $(n=1$, $0.4 \%)$, hypotension $(n=1,0.4 \%)$ and chest pain and reduced consciousness, requiring reversal agent $(n=1,0.4 \%)$. There were no significant bleeding or perforation reported for this cohort.

Conclusions Overall colonoscopy seems to be safe in the extreme ages with low complication rates. The caecal intubation rate was lower than the BSG/JAG recommended cut off $90 \%$, and this was mostly attributable to inadequate bowel preparation and obstructing lesions. If both of these limitations are adjusted for, the completion rate was $91.5 \%$. 